\title{
Scanning electron microscopic observation of Bruch's membrane with the osmium tetroxide treatment
}

\author{
TEIKO YAMAMOTO' AND HIDETOSHI YAMASHITA ${ }^{2}$ \\ From the 'Department of Ophthalmology, University of Tokyo School of Medicine Branch Hospital, 3-28-6 \\ Mejirodai, Bunkyo-ku, Tokyo 112, and the 'Department of Ophthalmology, University of Tokyo School of \\ Medicine, 7-3-1 Hongo, Bunkyo-ku, Tokyo 113, Japan
}

SUMMARY Scanning electron microscopic observation of Bruch's membrane was performed after removal of retinal pigment epithelium (RPE) with the osmium tetroxide treatment. Eight human eyes from subjects at various ages (from newborn to 77 years old) were examined in order to investigate aging changes in Bruch's membrane. The collagen fibres of the inner collagenous zone in young eyes formed a tightly interwoven membrane, and the meshes were regular and fine. In old eyes the meshes were irregular and coarse, and deposits were observed. Deposits were embedded in the collagen fibres of the inner collagenous zone, or attached to the surface on the innercollagenous-zone side of the basement membrane of RPE.

Bruch's membrane and its aging changes have been observed mainly by light and transmission electron microscopy (TEM) ${ }^{1-9}$ Scanning electron microscopic studies have been few, probably because it is technically difficult to remove retinal pigment epithelium (RPE) from Bruch's membrane without damage. Goldbaum and Madden digested RPE with trypsin and observed Bruch's membrane by scanning electron microscopy (SEM).$^{10}$ In the present study RPE was removed with the osmiun tetroxide treatment, ${ }^{11}$ and Bruch's membrane was observed by SEM. In addition, Bruch's membranes at various ages were examined in order to investigate aging changes.

\section{Material and methods}

One eye was obtained from each of eight patients. Table 1 lists the age and cause of donors' death or surgical enucleation. Six eyes were obtained at necropsy (cases 1-6). The eyes were enucleated and fixed in $2.5 \%$ glutaraldehyde 6 to 12 hours post mortem. The other two eyes (cases 7 and 8 ) were enucleated under general anaesthesia because of retinoblastoma and immediately fixed in $2.5 \%$ glutaraldehyde. The osmium tetroxide treatment was carried out according to the method of Komuro and Uehara. "After fixation in $2.5 \%$ glutaraldehyde the eyes were dissected into fragments of appropriate

Correspondence to Hidetoshi Yamashita MD. Department of Ophthalmology. University of Tokyo School of Medicine, 7-3-1 Hongo. Bunkyo-ku. Tokyo 113, Japan. size, and specimens containing macular regions were immersed in $2 \%$ osmium tetroxide solution, which was buffered to $\mathrm{pH} 7.4$ with $0 \cdot 1 \mathrm{M}$ cacodylate buffer, for 4 to 7 days at $4^{\circ} \mathrm{C}$. After this treatment the cells became fragile, so RPE cells were washed off Bruch's membrane with a jet stream of osmium tetroxide solution from a pipette.

For SEM the specimens were dehydrated with a graded series of ethanol, critical-point-dried, coated with platinum-vanadium, and observed by SEM (ISI-DS 130, Akashi, Japan). For TEM a part of each specimen was dehydrated with a graded series of ethanol and embedded in Epok 812 (Oken Shoji, Japan). Ultrathin sections were cut, stained with uranyl acetate and lead nitrate, and observed by TEM (JEM 1200EX).

Table 1 Eight eyes of eight individuals used in this study

\begin{tabular}{lll}
\hline Case & Age (years) & Comments \\
\hline Necropsied cases & & \\
1 & Newborn & Infantile sudden death \\
2 & 11 & Brain tumour \\
3 & 23 & Sudden death after delivery \\
4 & 56 & Cerebral haemorrhage \\
5 & 66 & Gastric cancer \\
6 & 77 & Cerebral infarction \\
Eyes surgically obtained & \\
7 & 0 & Retinoblastoma \\
8 & 2 & Retinoblastoma \\
\hline
\end{tabular}




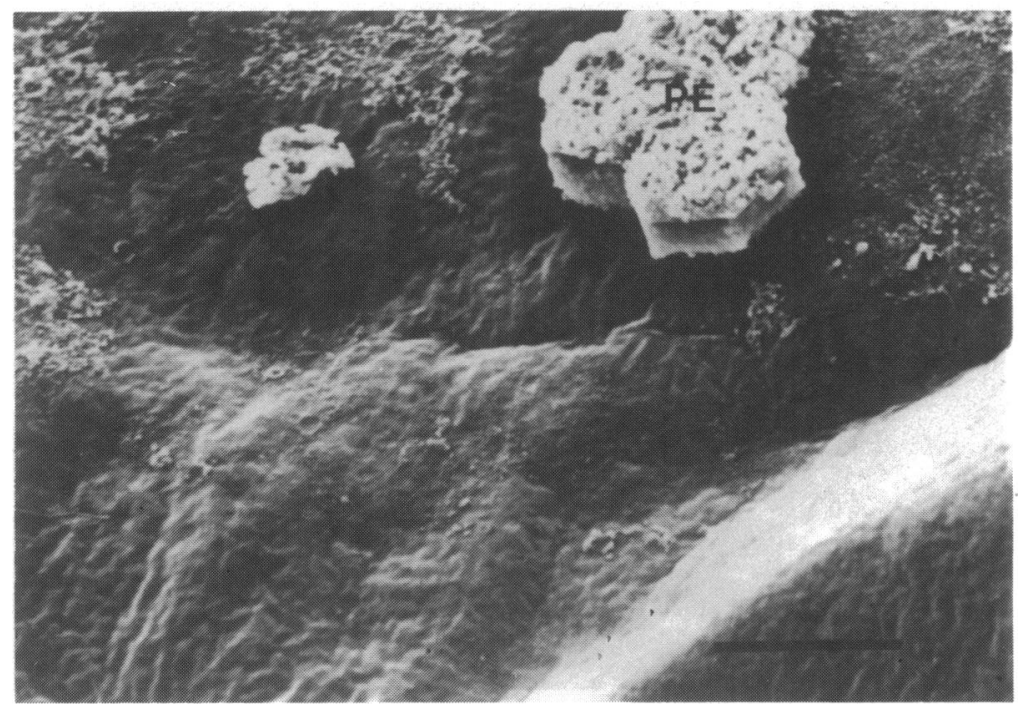

Fig. 1 Basement membrane of $R P E$ in a 23-year-old patient (case 3). PE: Patches of pigment epithelium remaining attached to Bruch's membrane. Bar $=10 \mu \mathrm{m}$.

\section{Results}

After the osmium tetroxide treatment the tissue, especially the cell components, became fragile and could be easily removed. As shown in Fig. 1, RPE cells were removed and the basement membrane of RPE cells was exposed. In some specimens the basement membrane of RPE was torn and the inner collagenous zone was exposed. The ground substance in the inner collagenous zone was washed off and the collagen fibre could be observed by SEM (Fig. 2).

In the Bruch's membrane of the young eyes (from the newborn to the 11-year-old patients) the collagen fibres in the inner collagenous zone formed a tightly interwoven membrane. The meshes were regular and fine. Deposits were absent in the inner collagenous zone on SEM observation (Figs. 2, 3). In Bruch's membrane of the old eyes (from the 56- to the 77year-old patients) the collagen fibres in the inner collagenous zone also formed an interwoven membrane, but the meshes were irregular and coarse, and the collagen fibres were inclined to aggregate and to form bundles in contrast to the young eyes (Figs. 4, $5,6)$.

Among the collagen fibres in the inner collagenous zone of the old eyes, deposits of various kinds were

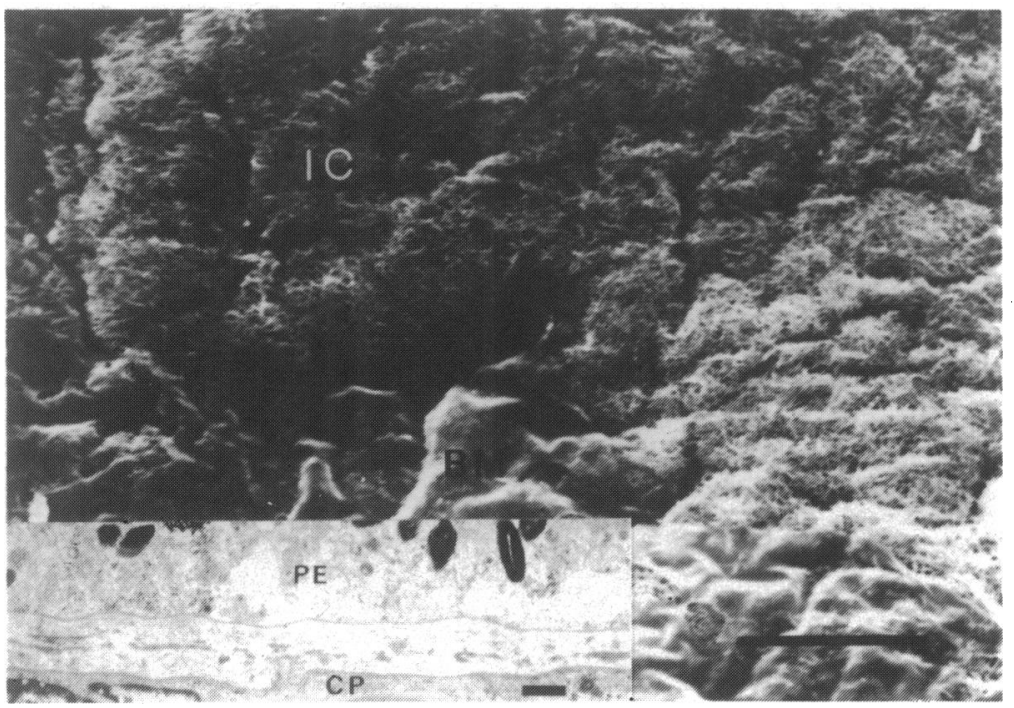

Fig. 2 Where basement

membrane of RPE (BM) is absent, the inner collagenous zone (IC) is exposed. The meshes of tight interweaving of collagen fibres are regular and fine. Deposits are absent. A newborn infant (case 1). Bar $=10 \mu \mathrm{m}$. Inset: Transmission electron micrograph of Bruch's membrane in same patient (case 1). Deposits are absent. PE: Retinal pigment epithelium. $\mathrm{CP}$ : choriocapillaris. Bar $=1 \mu \mathrm{m}$. 
Fig. 3 Inner collagenous zone in a 2-year-old patient (case 8). The meshes of tighly interweaving collagen fibres are regular and fine. Deposits are absent. BM: Basement membrane of RPE. Bar $=10 \mu \mathrm{m}$.

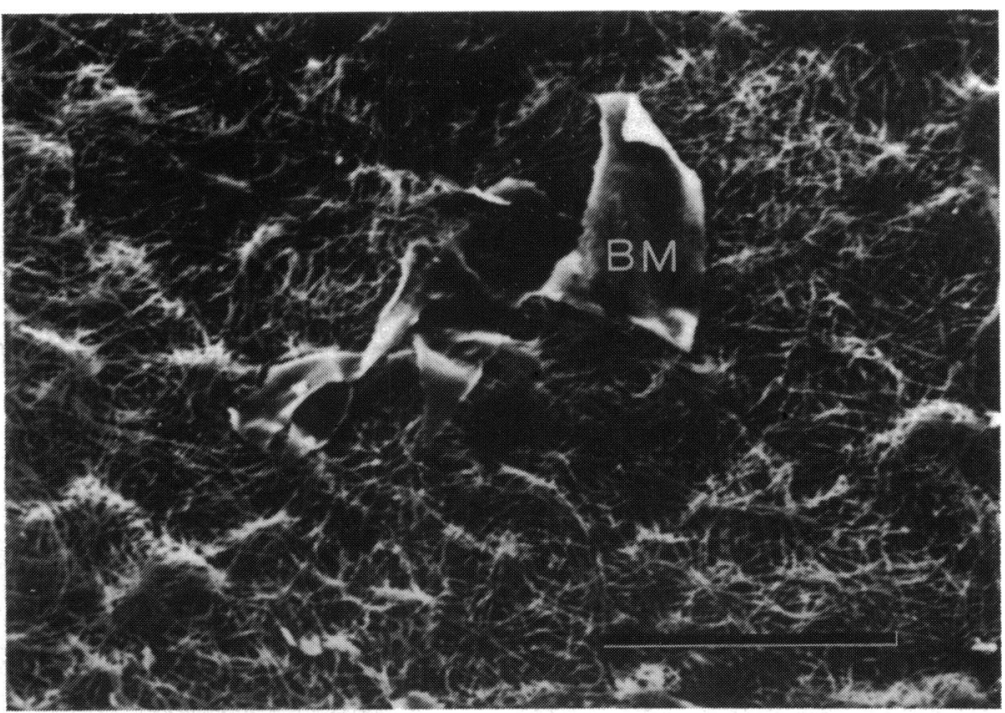

observed (Figs. 7, 8). As shown in Fig. 7, spherical structures and larger structures of other shapes were embedded in the collagen fibres. A mound-like structure, shown in Fig. 8, had a rather smooth surface and involved the surrounding collagen fibres. This was observed to project from the inner collagenous zone toward the RPE. Similar deposits were also observed to be attached to the surface on the innercollagenous-zone side of the basement membrane of RPE (Figs. 5, 6).

The results of the observations by SEM coincided with those by TEM, that is, deposits were absent in Bruch's membrane of the young eyes (Fig. 2, inset), and deposits were noted in the old eyes (Fig. 9). In Fig. 9 vesicular and tube-like structures, widespacing collagen, and so on were observed.

\section{Discussion}

With the osmium tetroxide treatment the tissues, especially the cell components, become fragile and can be easily removed. ${ }^{11}$ This technique is thought to be useful for removing the overlying cells and observing the underlying tissue. ${ }^{12}$ In this report Bruch's membrane was observed by SEM after removal of RPE. In some specimens the inner collagenous zone

Fig. 4 Inner collagenous zone in a 56-year-old patient (case 4). The meshes are irregular and coarse. The collagen fibres aggregate and form bundles. Bar $=10 \mu \mathrm{m}$.

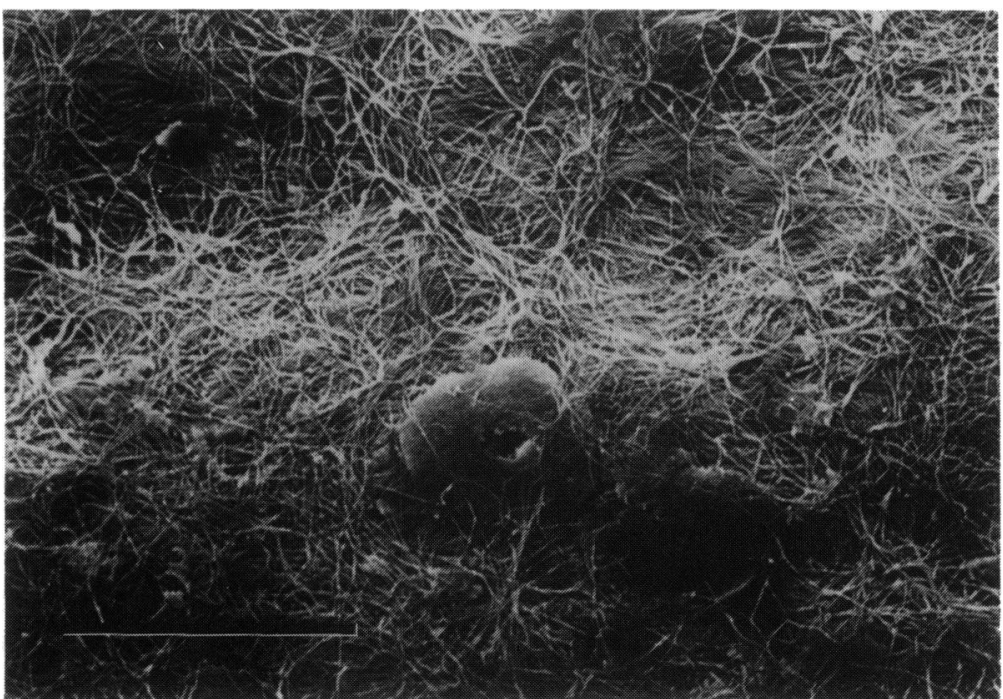




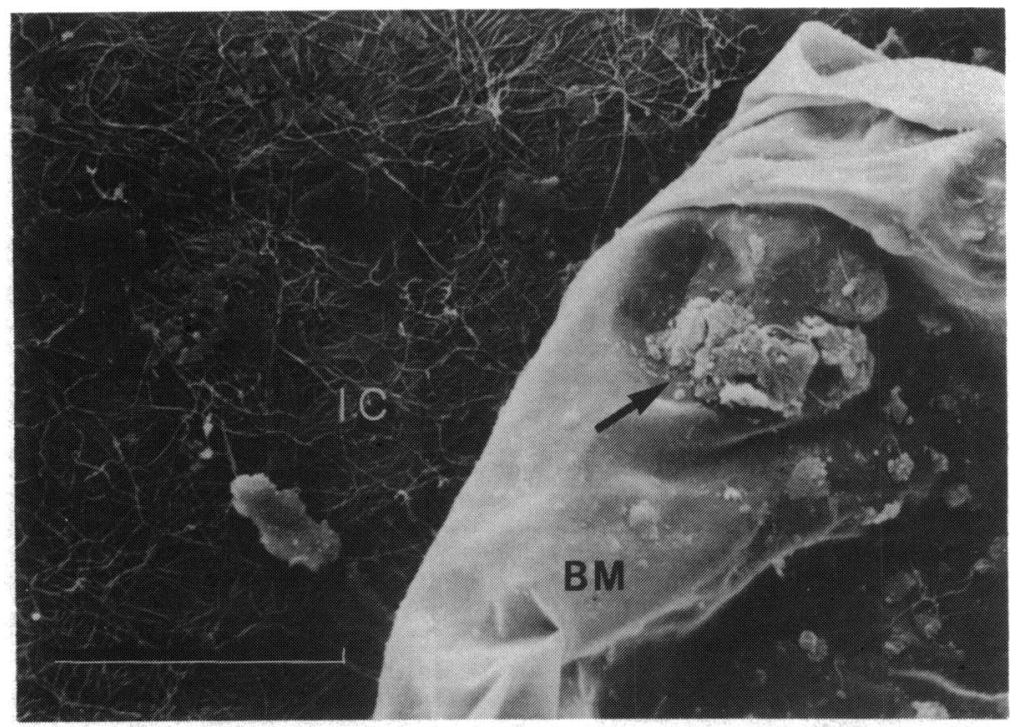

Fig. 5 The inner-collagenouszone-side surface of basement membrane of RPE (BM) and inner collagenous zone (IC) in case 4. Deposits are noted in the collagen fibres of IC and attach to $\mathrm{BM}$ (arrow). Bar $=10 \mu \mathrm{m}$.

was exposed because the basement membrane of the RPE was torn. Examination of Bruch's membrane by SEM after osmium tetroxide treatment showed that the meshes of a tightly interwoven membrane formed by the collagen fibres of the inner collagenous zone were regular and fine in the young eyes but were irregular and coarse in the old eyes. In the old eyes the collagen fibres in the inner collagenous zone tended to aggregate and to form bundles in contrast to those in the young eyes. The increase of collagen fibre cross-links was detected biochemically with increasing age in various organs. ${ }^{1314}$ The changes of the inner collagenous zone observed by SEM might be related to the aging of collagen of this type.

Deposits in the inner collagenous zone in the old eyes observed by SEM are thought to correspond to those observed by TEM. A mound-like structure (as shown in Fig. 8) was observed to project towards the RPE, and this is thought to correspond to a druse. While the deposits shown in Figs. 7 and 8 were embedded in the inner collagenous zone, the deposits shown in Figs. 5 and 6 were attached to the basement membrane of the RPE. Adhesion between the latter deposits and the basement membrane may be strong. The interpretation of this is obscure. Observations by TEM revealed that some deposits were located

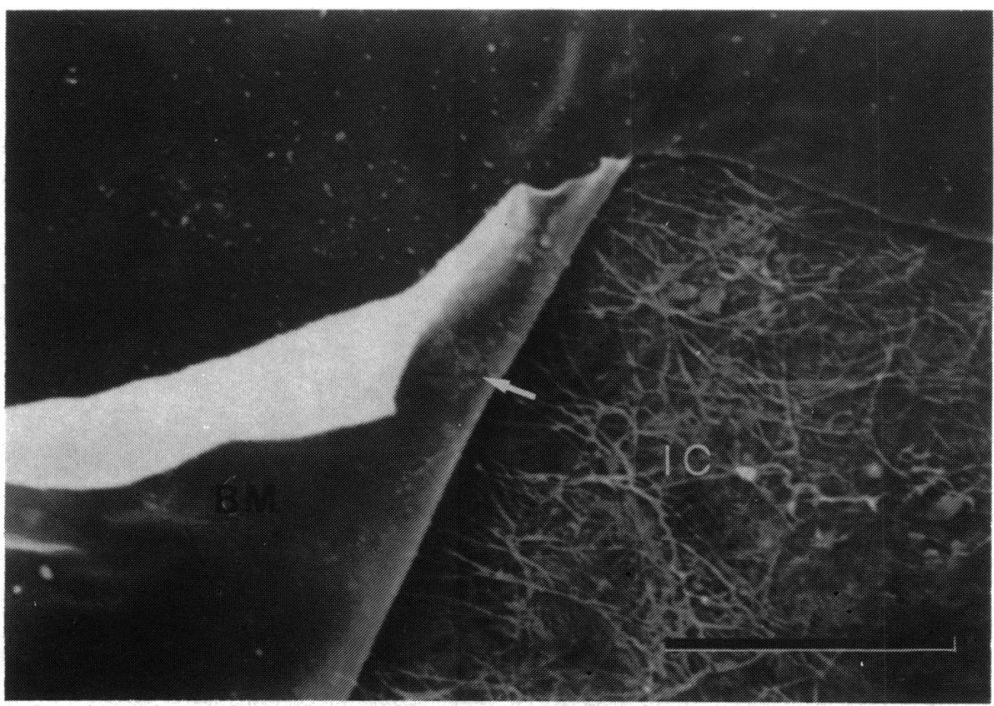

Fig. 6 Basement membrane of $R P E(\mathrm{BM})$ and inner collagenous zone (IC) in a 77-year-old patient (case 6). Deposits attached to the inner-collagenous-zone-side surface of basement membrane of RPE (arrow). Bar $=10 \mu \mathrm{m}$. 
Fig. 7 Inner collagenous zone in a 56-year-old patient (case 4). Deposits are noted among the collagen fibres. Bar $=1$ um.

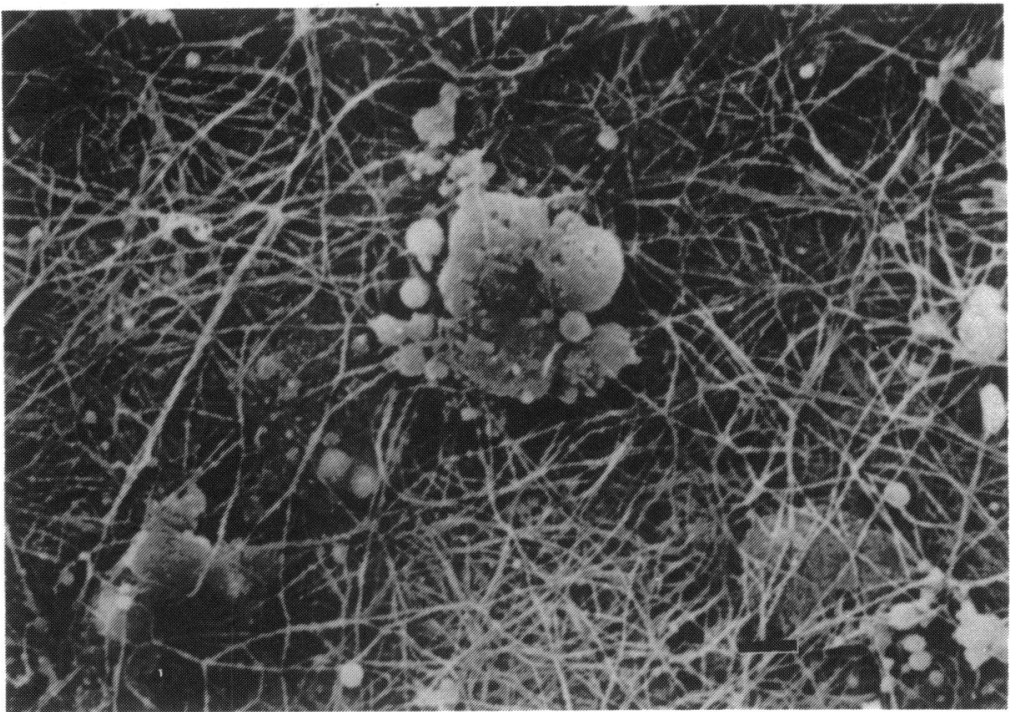

beneath the basement membrane of RPE. Some authors suggested that bulk release (apoptosis) of pigment epithelial cytoplasm leads to the formation of some deposits. ${ }^{579}$ The deposits shown in Figs. 5 and 6 , or at least some of them, may correspond to those.

The authors thank Drs Teiichi Yamamoto and Sadao Hori for helpful discussion.

\section{References}

1 Hogan MJ, Alvarado J. Studies on the human macula. IV. Aging changes in Bruch's membrane. Arch Ophthalmol 1967; 77: 410-20.
2 Farkas TG, Sylvester V, Archer D. The ultrastructure of drusen. Am J Ophthalmol 1971; 71: 1196-205.

3 Farkas TG, Sylvester V, Archer D, Altona M. The histochemistry of drusen. Am J Ophthalmol 1971; 71: 1206-15.

4 Hogan MJ. Role of the retinal pigment epithelium in macular disease. Ophthalmology 1972; 76: 64.

5 Burns R, Feeney-Burns L. Clinico-morphologic correlations of drusen of Bruch's membrane. Trans Am Ophthalmol Soc 1980; 78: 206-25.

6 Sarks SH, Van Driel D, Maxwell L, Killingsworth M. Softening of drusen and subretinal neovascularization. Trans Am Ophthalmol Soc UK 1980; 100: 414.

7 Feeney-Burns L, Ellersieck MR. Age-related changes in the ultrastructure of Bruch's membrane. Am J Ophthalmol 1985; 100: 686-97.

8 Marshall J, Laties A. The special pathology of the aging macula. In: Lavail M, Hollyfield JG, Anderson RE, eds. Retinal degen-
Fig. 8 Inner collagenous zone in case 4. A mound-like structure is noted (arrow). Bar $=1 \mu \mathrm{m}$.

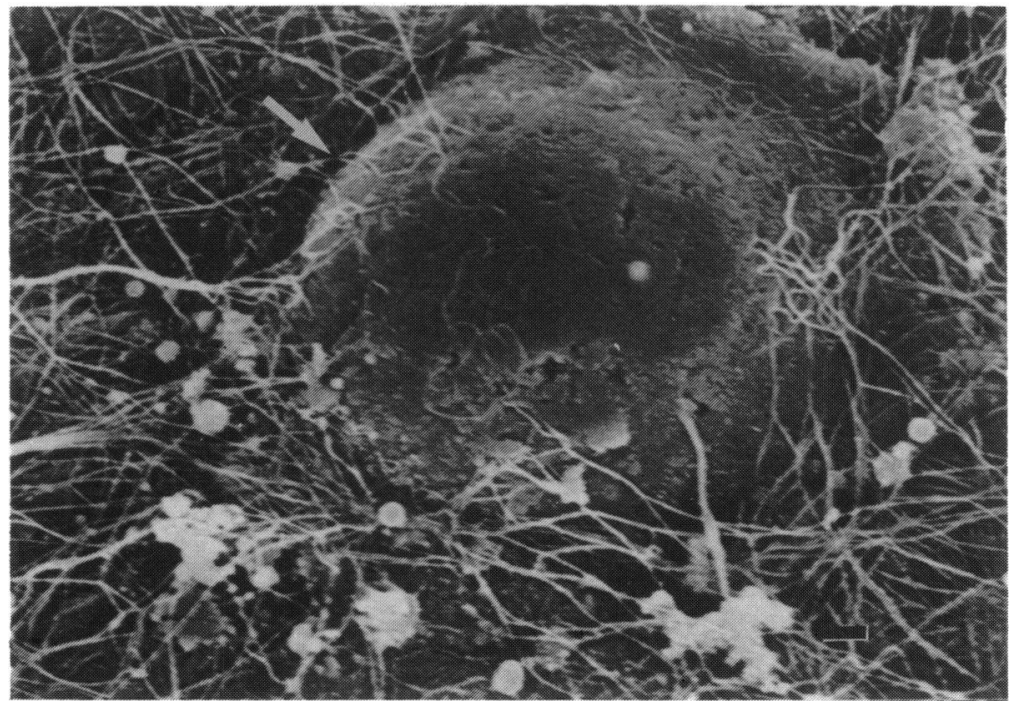




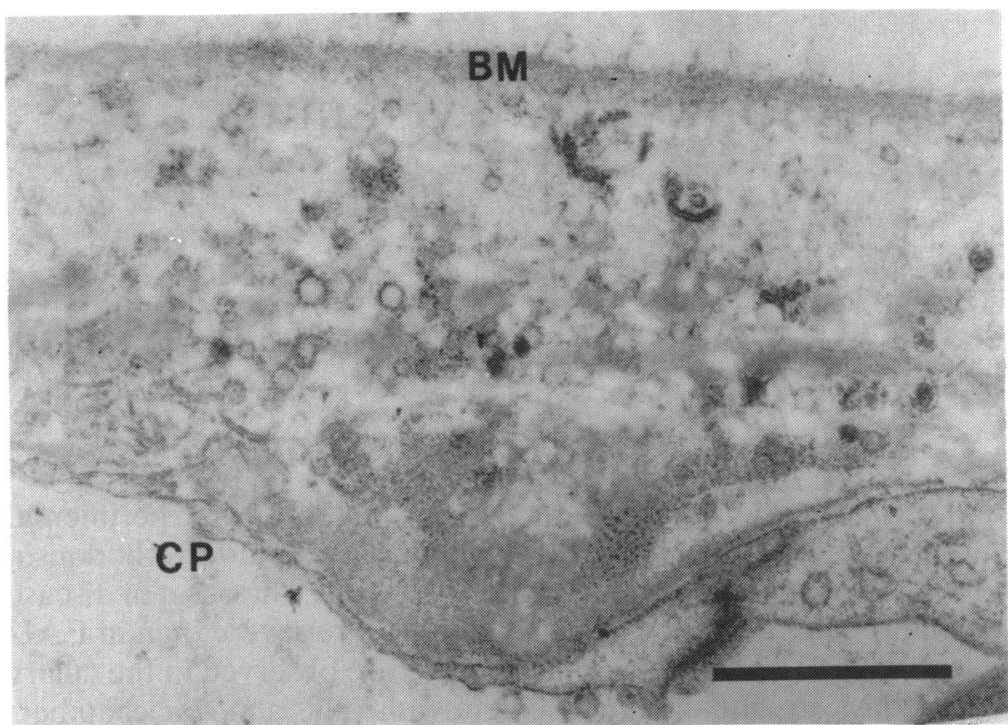

Fig. 9 Transmission electron micrograph of Bruch's membrane in case 4. Deposits are noted. BM: Basement membrane of RPE. CP. Choriocapillaris. Bar $=1 \mu \mathrm{m}$.

eration: experimental and clinical studies. New York: Liss, 1985: 389-400.

9 Feeney-Burns L, Gao CL, Tidwell M. Lysosomal enzyme cytochemistry of human RPE, Bruch's membrane and drusen. Invest Ophthalmol Vis Sci 1987; 28: 1138-47.

10 Goldbaum MJ, Madden K. A new perspective on Bruch's membrane and the retinal pigment epithelium. BrJ Ophthalmol 1982; 66: 17-25.

11 Komuro J, Uehara Y. Application of SEM-maceration (fenestration of the basal lamina of intestinal villi). Taisha (Metabolism) 1986; 23 (5): i-ii.
12 Yamashita H. Scanning electron microscopic observation of ciliary pigment epithelium. Folia Ophthalmol Jpn 1987; 38: 1713-6.

13 Schaub MC. Qualitative and quantitative changes of collagen in parenchymatous organs of the rat during aging. Gerontologia 1963; 8: 114-22.

14 Zwolinski RC, Hamlin R, Kohn RR. Age related alteration in human heart collagen. Proc Soc Exp Biol Med 1976; 152: 362-5.

Accepted for publication 3 March 1988. 